\title{
Optical and Optoelectronic Properties of ZnS Nanostructured Thin Film
}

\author{
J.P. BORAH* AND K.C. SARMA \\ Department of Instrumentation and USIC, Guwahati University \\ Guwahati, Assam, 781014, India
}

(Received April 3, 2008)

\begin{abstract}
ZnS nanocrystalline thin films were grown into the polyvinyl alcohol matrix and were synthesized by chemical route. Films were prepared on glass substrate by varying the deposition parameters and $\mathrm{pH}$ of the solution. Nanocrystalline thin film prepared under optimum growth conditions shows band gap value $3.88 \mathrm{eV}$ as observed from optical absorption data. The band gap is found to be higher $(3.88 \mathrm{eV})$ indicating blue shift. The particle size, calculated from the shift of direct band gap, due to quantum confinement effect is $5.8 \mathrm{~nm}$. Photoluminescence spectrum shows the blue luminescence peaks (centered at $425 \mathrm{~nm}$ ), which can be attributed to the recombination of the defect states. ZnS nanocrystalline thin films are also found to be photosensitive in nature. However, the photosensitivity decreases due to ageing and exposure to oxygen. In case of nanostructured film, the $I-V$ characteristics are observed in dark and under illumination showing photosensitive nature of these films, too. The dark current, however, is found to be greater when observed in vacuum compared to air. Both dark current and photocurrent are found to be ohmic in nature up to a certain applied bias. The observed data shows that nanostructured films are found to be suitable for device application. The surface morphology of the film is also characterized by scanning electron microscope.
\end{abstract}

PACS numbers: 73.61.Ga, 73.61.Tm, 74.25.Gz

\section{Introduction}

Semiconductor nanoparticles, which have changed properties resulting from quantum confinement, have drawn considerable interest and are currently being investigated $[1,2]$. Semiconductor nanoparticles exhibit size-dependent electronic band gap energies [3], melting temperatures [4], solid-solid phase transition temperatures [5] and pressures [6]. In addition to these, doped semiconductor nanoparticles have tremendous potential for use in light emitting applications. These

*corresponding author; e-mail: jpb@iitg.ernet.in 
properties of nanocrystals make them an interesting category of material for optoelectronic applications. These nanoparticles may find applications in nonlinear optical devices, photocatalysis, etc. II-VI compound semiconductors have immense technological importance in different applied branches of science and technology. Zinc sulphide $(\mathrm{ZnS})$ is an important II-VI semiconductor material with a wide direct band gap $E_{\mathrm{g}}=3.68 \mathrm{eV}$ (bulk) [7]. $\mathrm{ZnS}$ has been studied due to its wide applications as phosphors and catalysts [8]. $\mathrm{ZnS}$ is also applicable for a variety of other applications such as electro-luminescent devices, solar cells, and many other optoelectronic devices. ZnS has a low exciton Bohr radius $(2.5 \mathrm{~nm}$ ) that makes its nanoparticles interesting as small biomolecular probes for fluorescence and laser scanning microscopy. ZnS is also currently used as a shell or capping layer in core/shell nanoprobes such as CdSe/ZnS core/shell structures [9].

In this paper we report some optical and optoelectronic properties of $\mathrm{ZnS}$ nanocrystalline thin films, prepared by chemical method.

\section{Experimental}

\subsection{Preparation technique}

For preparing nanocrystal of ZnS, the matrix of polyvinyl alcohol (PVA) is prepared by stirring for $3 \mathrm{~h}$ with temperature controlled magnetic stirrer. The matrix is mixed with the reactants zinc chloride $\left(\mathrm{ZnCl}_{2}\right)$ solution and then again stirred. As soon as the nanostructure is formed it immediately enters into the gap and can neither come out nor can enhance in size. The particle size is controlled by varying temperature of the reaction, amount of reactant, stirring rate, and $\mathrm{pH}$ of the solution. The film over glass substrate contains the nanocrystalline of $\mathrm{ZnS}$ specimen embedded in PVA matrix.

\subsection{Measurement technique}

The electrical conductivity was measured with coplanar high purity silver electrodes as ohmic contact to these films. The electrodes were separated by a gap of $1 \mathrm{~mm}, 2 \mathrm{~mm}, 5 \mathrm{~mm}$, printed on the film surface by conductive silver paint (Micro tip, CW 2200 MTP, UK). To avoid the effect of silver diffusion into the films, silver paint was applied on the film surface after annealing and just before conductivity measurement. In case of nanocrystalline $\mathrm{ZnS}$ film in PVA matrix, conducting silver paste of Maswin India mixed with some thinner were used. In all the conductivity measurement two probe methods were adopted. The optical absorbance of films were recorded at room temperature using double beam automated spectrophotometer (Hitachi U3210). Photoluminescence (PL) spectra were recorded using (Thermospectronic AMINCO) luminescence spectrometer. Thickness of the film was measured by gravimetric method using highly sensitive electronic Microbalance (AB54-S, METTLER TOLEDO) $I-V$ characteristic is recorded using Keithley 6514 system electrometer and with Hioki 7020 variable power supply. For creating vacuum, rotary vacuum pump is used. 


\section{Result and discussion}

The optical properties of ZnS nanocrystalline thin films are determined from absorbance measurements in the range of $200-500 \mathrm{~nm}$. Figure 1 shows the absorption spectra of nanocrystalline ZnS thin films embedded in PVA matrices.

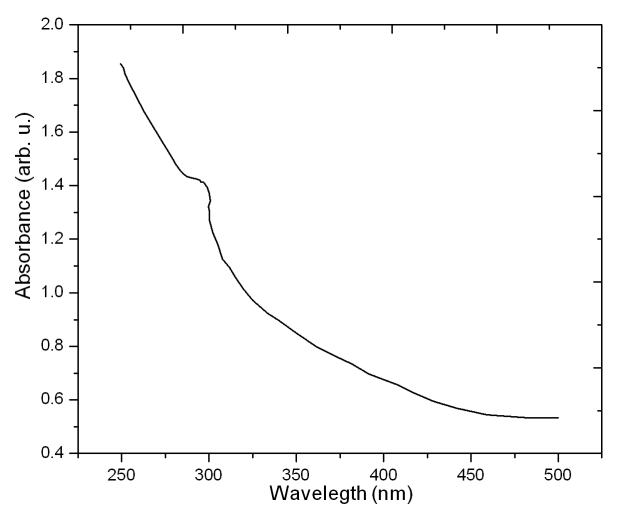

Fig. 1. Absorbance vs. wavelength plot of nanocrystalline ZnS/PVA.

Absorption coefficient $(\alpha)$ associated with the strong absorption region of the films was calculated from absorbance $(A)$ and the film thickness $(t)$ using the relation [10]:

$$
\alpha=2.3026 \mathrm{~A} / \mathrm{t} \text {. }
$$

The absorption coefficient $(\alpha)$ was analyzed using the following expression for near-edge optical absorption of semiconductors $(\alpha h \nu)=K\left(h \nu-E_{\mathrm{g}}\right)^{n / 2}$ where $K$ is constant, $E_{\mathrm{g}}$ is the separation between the valence and conduction bands and $n$ is a constant that is equal to 1 for direct band gap semiconductors. The band gap values were determined from the intercept of the straight-line portion of the $(\alpha h \nu)^{2}$ against the $h \nu$ graph on the $h \nu$-axis using computer fitting program (Fig. 2).

The linear part shows that the mode of transition in these films is of direct nature. The calculated band-gap value of the film was $3.88 \mathrm{eV}$. The band-gap values are higher than bulk value of $\mathrm{ZnS}(3.68 \mathrm{eV})$ because of quantum confinement of ZnS nanocrystals. The absorption spectra (Fig. 1) reveal that the excitonic absorption peaks for nanocrystal are blue shifted compared to the bulk band gap (3.68 eV corresponding to the absorption edge at $336 \mathrm{~nm}$ ) and clearly indicate strong quantum size effect. Size quantization of carriers in a small volume crystallite is well known to cause the blue shift. The shift of band gap might also be utilized in determining the crystal radius $(r)$ using the effective mass approximation relation $[11,12]$ :

$$
\Delta E_{\mathrm{g}}=E_{\mathrm{g}}(\text { film })-E_{\mathrm{g}}(\text { bulk })=h^{2} / 8 \mu r^{2}-1.8 e^{2} / \epsilon_{0} \epsilon_{\mathrm{r}} r,
$$




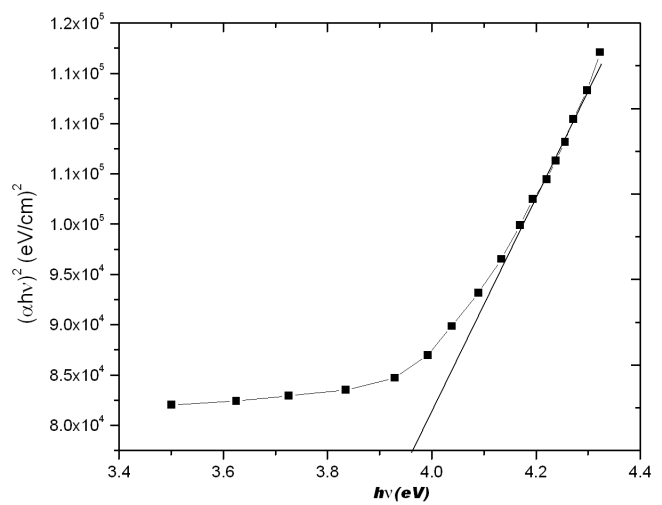

Fig. 2. Plot to determine the direct band gap of ZnS/PVA nanocrystalline thin films.

where $1 / \mu=1 / m_{\mathrm{e}}^{*}+1 / m_{\mathrm{h}}^{*}$ is the reduced mass of electron hole effective mass, $m_{\mathrm{e}}^{*}=0.34 m_{0}$ and $m_{\mathrm{h}}^{*}=0.23 m_{0}$ and $\epsilon_{\mathrm{r}}=8.76$ is the permittivity of the sample. The particle size obtained from this sample is $5.86 \mathrm{~nm}$.

Photoluminescence spectra measured at room temperature $(300 \mathrm{~K})$ of the nanocrystalline ZnS/PVA thin film deposited on glass surface are shown in Fig. 3.

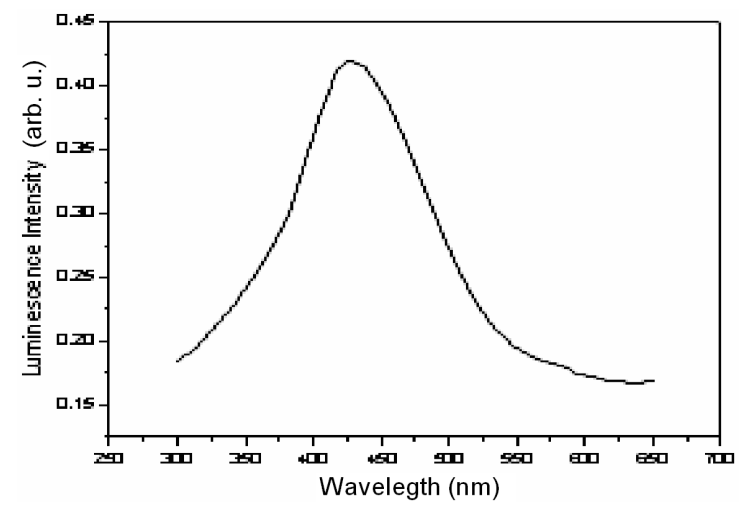

Fig. 3. PL spectrum of ZnS/PVA nanostructured film.

The samples are photoexcited at $300 \mathrm{~nm}$. The plot contains the peak centered at $425 \mathrm{~nm}$. The PL peak at $425 \mathrm{~nm}$ has been known due to the recombination between the sulfur-vacancy-related donor and the valence band [13]. The sulfur deficiency in the synthesized nanocrystalline ZnS/PVA thin film was confirmed from energy distribution of X-ray (EDX) measurement of the sample (Fig. 4).

$I-V$ characteristics of nanostructured $\mathrm{ZnS}$ films are shown in Fig. 5. Both dark current and current under illumination increase linearly for both positive and negative applied bias up to $\pm 100 \mathrm{~V}$. However, when observations are made in vacuum, the dark current increases due to decrease in resistance (Fig. 6). 


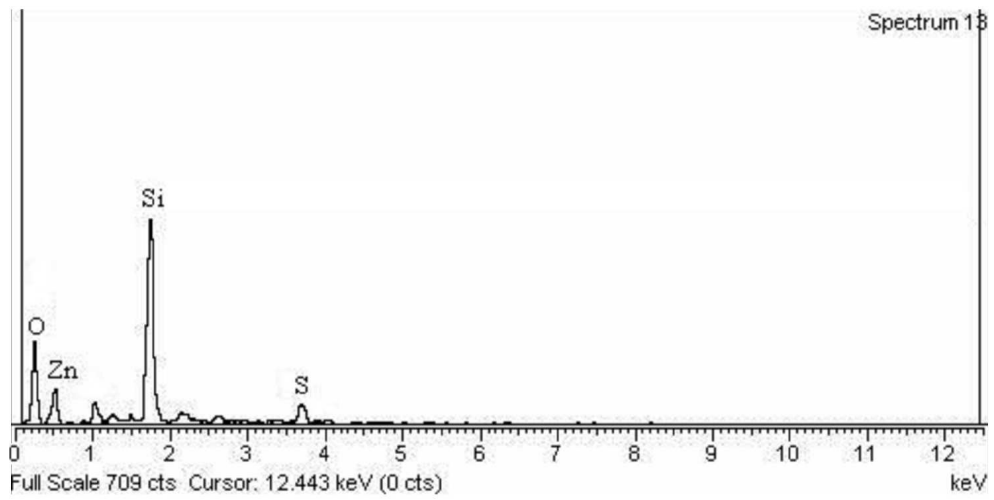

Fig. 4. EDX spectrum of ZnS/PVA nanostructured film.

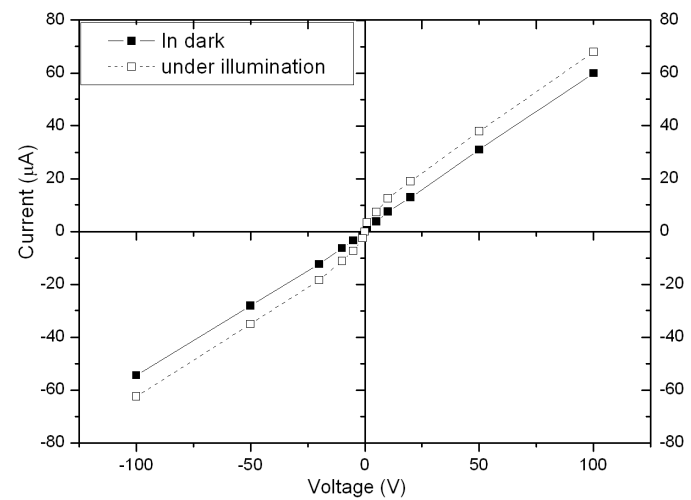

Fig. 5. Currect vs. voltage plots in dark and under illumination for a ZnS/PVA nanostructured film.

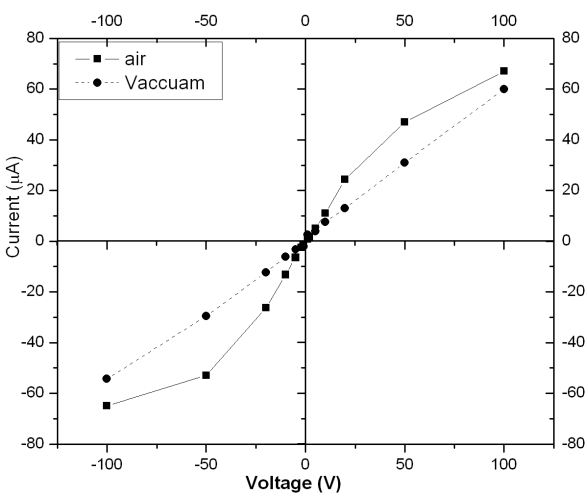

Fig. 6. Current vs. voltage plots in dark for a ZnS/PVA nanostructured film in air and vacuum. 
In air, the dark current decreases due to increase in resistance. The resistance variation in air is attributed due to chemiadsorption of oxygen.

The chemiadsorption of oxygen in air is supported by $\mathrm{ZnO}$ peaks observed in X-ray diffraction (XRD) spectra (not shown here). It is generally accepted that oxygen is chemiadsorbed at a surface site such as sulphur vacancy in the form of an ionized oxygen atom or molecule, i.e. $\mathrm{O}_{2}^{-}$or $\mathrm{O}^{-}$. This results in a reduced concentration of free electrons at the surface and the observed reduction in the conductivity or dark current [14].

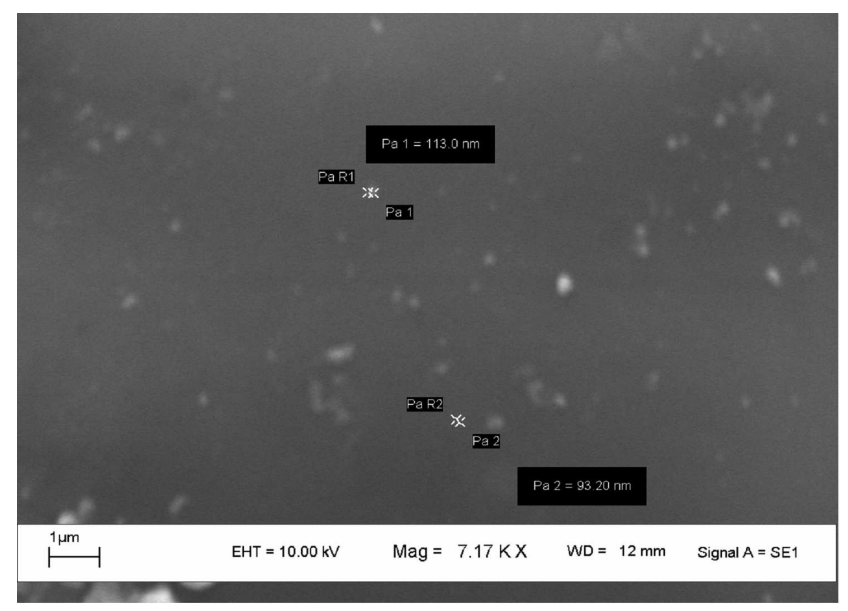

Fig. 7. Scanning electron micrograph of ZnS/PVA nanostructured thin film.

Figure 7 shows the scanning electron microscopy (SEM) picture of ZnS nanocrystalline thin film in optimum growth condition. SEM images indicate that as-synthesized thin film consists of $\mathrm{ZnS}$ nanoparticle of spherical shape. Figure 5 also shows that the surface of the film is smooth, uniform and no crack could be observed. The particle size from the SEM is found to be in the range of 92-114 nm. The size of the particle is increased due to the agglomeration of the nanoparticle.

\section{Conclusion}

ZnS nanocrystalline films grown on glass substrates using PVA as matrix are also found to be photosensitive. The band gap of nanocrystalline $\mathrm{ZnS}$ increases to higher value as observed from optical absorption data. The increase in band gap value to $3.88 \mathrm{eV}$ suggests decrease in crystallite size and formation of quantum dots in PVA matrix. Films kept in air adsorb oxygen from air and their resistance increases as observed from $I-V$ characteristics. SEM study indicated a nanoparticle formation in film. 


\section{Acknowledgments}

The authors would like to acknowledge Physics Dept and CIF, IITG for providing XRD, SEM, and PL facilities. We would also like to acknowledge Department of Chemistry, Gauhati University, for spectrophotometer.

\section{References}

[1] L.E. Brus, J. Phys. Chem. 90, 2555 (1986).

[2] Y. Wang, N. Herron, J. Phys. Chem. 95, 525 (1991).

[3] L.E. Brus, Chem. Phys. 80, 4403 (1984).

[4] A.N. Goldstein, C.M. Echer, A.P. Alivisatos, Science 356, 1425 (1992).

[5] S.B. Qadri, E.F. Skelton, D. Hsu, A.D. Dinsmore, J. Yang, H.F. Gray, B.R. Ratna, Phys. Rev. B 60, 9191 (1999).

[6] C.C. Chen, A.B. Herhold, C.S. Johnson, A.P. Alivisatos, Science 276, 398 (1997).

[7] K. Sooklal, B.S. Cullumn, S.M. Angel, C.J. Murphy, J. Phys. Chem. 100, 4551 (1996).

[8] Y.C. Zhu, Y. Bondo, D.F. Xue, Appl. Phys. Lett. 82, 1769 (2003).

[9] A. Thakur, C. Fradin, Can. Undergraduate Phys. J. 3, 7 (2005).

[10] R.S. Longhurst, Geometrical and Physical Optics, Longmans Green, London 1957.

[11] A.D. Yoffe, Adv. Phys. 42, 173 (1993).

[12] Y.S. Yuang, F.Y. Chen, Y.Y. Lee, C.L. Liu, Jpn. J. Appl. Phys. 76, 304 (1994).

[13] S. Lee, D. Song, D. Kim, J. Lee, S. Kim, I.Y. Park, Y.D. Choi, Mater. Lett. 58, 342 (2004).

[14] G. South, M. Hughes, Thin Solid Films 20, 135 (1974). 